\title{
About fast and slow charge packets in polymeric materials under DC stress
}

\author{
Davide Fabiani, Gian Carlo Montanari \\ Department of Electrical Engineering, University of Bologna \\ Bologna, Italy \\ Leonard A. Dissado \\ Department of Engineering, University of Leicester \\ Leicester, United Kingdom \\ Christian Laurent, Gilbert Teyssedre \\ LAPLACE - Université Paul Sabatier, \\ Toulouse, France
}

\begin{abstract}
The presence of slow space charge packets crossing the insulation thickness from one electrode to the other and causing significant electrical field distortion has been reported already in several papers. They are activated in general by very high DC fields or, in highly polluted materials, by relatively low fields and constitute an important ageing factor, concerning DC electrical stress. It has been observed, in fact, that such packets can cause accelerated breakdown of insulation.

The development of fast systems for space charge measurements has allowed the presence of almost instant heterocharge to be observed close to electrodes in certain field and temperature conditions, especially in cable models. This has been explained often by the separation of ionic charge populations, even though such heterocharge appears also in materials, such as Polyethylene or cross-linked Polyethylene that represent the best extra-clean technologies. The measurements reported here use a high speed technique to investigate the build up of heterocharge in model cables that have been treated to remove volatile chemical species. They show that in fact the heterocharge is built up by many very small and very fast charge packets (i.e. charge packets having a high mobility), which are injected from both electrodes and cross the insulation in less than one second. Because the packet charge is unable to exit the counter-electrode at the same rate at which it arrives, hetero-charge is built up within just a few seconds from the beginning of the polarization. The mobility of these charges, depending significantly on temperature, is estimated through observation of charge packets as a function of time, and compared with that of the already-known slow packets, generally occurring at higher fields with respect to fast packets. The basis for the interpretation and modelling of such phenomena is discussed.
\end{abstract}

Index Terms — Space charge, fast packets, slow packets, HVDC, XLPE.

\section{INTRODUCTION}

SPACE charge accumulation is known to play a very important role in the aging and breakdown of HVDC solid polymeric insulation, due to local electric field enhancement. If the applied field exceeds the threshold for space charge accumulation, charge injected from the electrodes can accumulate in traps located at the interface with electrodes and in the insulation bulk [1-4]. Buildup 
of injected charge can be observed often close to the injecting electrode, forming a homocharge distribution after relatively short times of voltage application, depending on electric field and temperature. At other times, heterocharge is seen to accumulate close to electrodes. Models based on charge injection via a Schottky barrier, transport via either two levels of traps or a continuous exponential trap distribution, and presence or absence of an extraction barrier are able to reproduce many of the main features of space charge and conduction current that have been observed, though the details cannot yet be fully simulated [5-9].

Charge packets are another feature associated with charge injection and transport which has been observed in solid polymeric insulation. They are activated generally by high DC fields, with inception values depending on material (lowered by the presence of contaminants), temperature and mechanical stress [10-14]. They have typically mobility in the range $10^{-14}$ to $10^{-12} \mathrm{~m}^{2} \mathrm{~V}^{-1} \mathrm{~s}^{-1}$, and for this reason they are named in the following as "slow" packets. Charge packets constitute an important ageing factor, concerning DC electrical stress, due to large magnification of the electrical field inside the insulation associated with packet movement. It has been observed, in fact, that such packets can cause accelerated breakdown of insulation $[15,16]$, particularly when they are associated with a continual charge accumulation [17]. Various theories have been proposed to explain the generation of slow packets, from hysteresis of the fielddependence of the injection current, or fluctuations in the current injected from electrodes, to field-induced ionization of impurities or neutral sites [15, 18-21]. Only one of these theories has reached the stage of a successful simulation [21], where the creation and transport of large slow negative packets was described by assuming field ionization in a three-level system comprising an ionizable state, trap state, and valence band. In this case a plane of negative ionization is transferred through the system as mobile holes released by the high field at the front of the plane move in the opposite direction to the plane and recombine with the ionized states at the rear of the plane. Simulations of the model for space charge accumulation described in [8] also gave a single charge packet occasionally that was related to fluctuations in the injection process. However, none of these theories or simulations is able to describe the large generality of cases of slow charge packets. The common point is that a very high electrical field is needed to activate the packets. The type of charge carriers, the field distribution at the packet edges, the effect of electrodes, contaminants, and temperature are still open to different and contrasting interpretations.

A point that has not been observed or commented upon yet, mainly because the measurement systems for space charge detection are not fast enough, is that heterocharge formation, which seems often the start up of slow packets, may occur very rapidly, i.e. fraction of a second or a few seconds. This is the case of space charge measurements performed on HV model cables discussed in this paper. A significant accumulation of heterocharge close to both electrodes (semicon layers) was observed from several tests carried out in different laboratories involved in the European project HVDC [9, 22]. More accurate and fast measurements show that such charge forms just a few seconds or fraction of seconds after voltage application. This rapid appearance of heterocharge might be explained through the separation of ionic species, e.g. due to the presence of contaminants and/or crosslinking by-products in the insulation bulk. However, as shown in [23], heterocharge accumulation could be associated also with injection, when the rate of charge trapping is low compared to the transit time of the charge and extraction is slow compared to the rate of arrival of charge (i.e., in the presence of an extraction barrier). In this case a heterocharge region would build up at the counter electrode, with a width that increases with time but without any observable charge in the bulk of the material [23]. In order to understand the nature and dynamics of this fast-accumulating heterocharge, investigations have been performed by carrying out space charge tests using an ultra fast acquisition system. The results of such investigation are reported in the following.

\section{TEST PROCEDURES}

The specimens considered in this paper consist of cable models reproducing $\mathrm{HV}$ cables on a reduced-scale (minicables $)$, made by three layers: inner semicon $(0.7 \mathrm{~mm}$ thick), insulation layer of XLPE material (1.5 mm thick) and outer semicon $(0.15 \mathrm{~mm}$ thick $)$. The conductor diameter is $2.8 \mathrm{~mm}$ (Fig. 1).

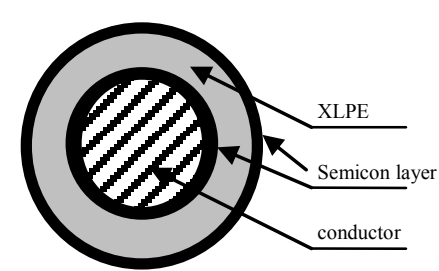

Figure 1. Sketch of tested minicable. Conductor diameter $=2.8 \mathrm{~mm}$; Inner semicon thickness $=0.7 \mathrm{~mm}$; dielectric $(\mathrm{XLPE})$ thickness $=1.5$ $\mathrm{mm}$. Outer semicon $=0.15 \mathrm{~mm}$.

Specimens were thermally treated for 5 days at $80{ }^{\circ} \mathrm{C}$ in order to expel most of the cross-linking by-products and contaminants. Space charge measurements were carried out through the Pulsed Electro Acoustic (PEA) technique at field values exceeding the threshold for space charge accumulation, i.e., $60 \mathrm{kV}$, which corresponds to a mean field of about $40 \mathrm{kV} / \mathrm{mm}$ (the threshold is around 10 $\mathrm{kV} / \mathrm{mm}$ at $25^{\circ} \mathrm{C}$ ). The poling time was set in two ways. Tests were performed in order that charge could reach the steady-state condition (usually $10000 \mathrm{~s}$ are enough). Other tests, mostly addressed to detecting and understanding fast space charge formation phenomena, were lasting less than $100 \mathrm{~s}$ since attention was paid to the charge dynamics in the first few seconds of polarization. The effect of temperature on charge dynamics was investigated by carrying out tests in an oven under 
isothermal conditions (no temperature gradient applied to insulation) at $35^{\circ} \mathrm{C}, 45^{\circ} \mathrm{C}$ and $70^{\circ} \mathrm{C}$.

In order to record space charge profiles at times as close together as possible, e.g. every 10-100 ms, with minimum noise, an average on at least 100 signals was performed. A Digital Signal Averager was used for this purpose. This device, in fact, thanks to its performing processor and a fast connection to the $\mathrm{PC}$, is able to acquire, average, and store signals at a rate that can reach some $\mathrm{MHz}$, thus enabling the fastest acquisition of space charge profiles. Space charge profiles can be thus displayed every $0.05 \mathrm{~s}$, with each profile an average of 100 acquired PEA signals.

\section{TEST RESULTS}

Space charge patterns obtained from PEA tests on minicables using a standard acquisition system are reported in Figs. 2 and 3. Tests are relevant to measurements at a poling voltage of $60 \mathrm{kV}$ applied for $10000 \mathrm{~s}$ (depolarization of $3600 \mathrm{~s}$ ), at a temperature of $25^{\circ} \mathrm{C}$ (Fig. $2 \mathrm{~A}$ and $3 \mathrm{~A}, \mathrm{~B}$ ) and $70{ }^{\circ} \mathrm{C}$ (Fig. 2B), carried out on XLPE mini-cables $[9,22]$. The difference between the two figures is in the materials. While the one of Fig. 2 is the reference for high voltage cables, the XLPE of Fig. 3 is manufactured mostly for MV cables, untreated (Fig. 3B) or thermally treated for 5 days at $80{ }^{\circ} \mathrm{C}$ (Fig. 3A).

As can be seen, heterocharge builds up close to both electrodes, particularly at high temperature, almost immediately at the beginning of polarization. At room temperature, and, particularly, in the untreated MV-grade material, slow packets of negative/positive charge can be observed moving across the insulation bulk. We must remember that the PEA signal is sensitive to the net charge at each point of the insulation bulk. Hence, if positive and negative charge packets having similar amplitude and injected simultaneously from both electrodes overlap at a given point, the detected PEA profile could show up as if no charge was accumulated at that particular point. It can be easily understood that this problem makes the interpretation of the space charge profiles detected from measurements more complicated. In particular, the separation of positive and negative charge packet contributions could be very difficult.

The dynamic of charge packets can be inferred by means of the results of PEA measurements performed on the same XLPE mini-cables using the fast acquisition system described in the previous Section. These measurements yield space charge profiles such as those reported in Figures 4 and 5. The absolute time, i.e., the time relevant to the acquisition from the beginning of the polarization, is indicated on the bottom of each profile.

Figure 4 shows charge profiles obtained at $35^{\circ} \mathrm{C}$ under a poling voltage of $60 \mathrm{kV}$, at different poling times (from 0 to $0.9 \mathrm{~s})$. As can be seen, at the beginning of polarization no space charge is evident (Fig. 4A). After $0.1 \mathrm{~s}$ a small packet of positive charge seems to be injected from the anode (circled in Fig. 4B). This charge moves, then, following the direction indicated by the arrow. At time $\mathrm{t}=0.3 \mathrm{~s}$ it is located around the middle of the insulation bulk (see Fig. 4C) and reaches the counter electrode at $\mathrm{t}=0.5 \mathrm{~s}$ (see Fig. 4D). An example of a negative charge packet crossing the insulation bulk is reported in Figs. 4E-H. In this case the negative charge, indicated by a circle, moves from cathode to anode in about 0.3 s. A simultaneous transit of positive and negative packets has been often observed in space charge profiles. For the sake of clarity here we chose profiles showing separately positive and negative packets.

The charge packets detected in these profiles are quite small and difficult to separate from the background. In order to show them more clearly, first we have subtracted the profile obtained at the beginning of the experiment where no space charge exists. Then the profiles were processed by means of the Stationary Wavelet Transform (SWT) to reduce the background noise. The results are given in Fig. 5, for the positive (Fig. 5A) and negative (Fig. 5B) charge packets. They show more clearly the existence of small positive and negative packets crossing the insulation. Increasing the temperature to $45{ }^{\circ} \mathrm{C}$, positive and negative packets are seen that are faster than at $35{ }^{\circ} \mathrm{C}$. They cross the insulation thickness in $0.3 \mathrm{~s}$ for the positive packets and $0.2 \mathrm{~s}$ for the negative ones. The effect is strengthened at $70{ }^{\circ} \mathrm{C}$, where the positive charge crosses the insulation in $0.15 \mathrm{~s}$ and the negative in less then $0.05 \mathrm{~s}$.

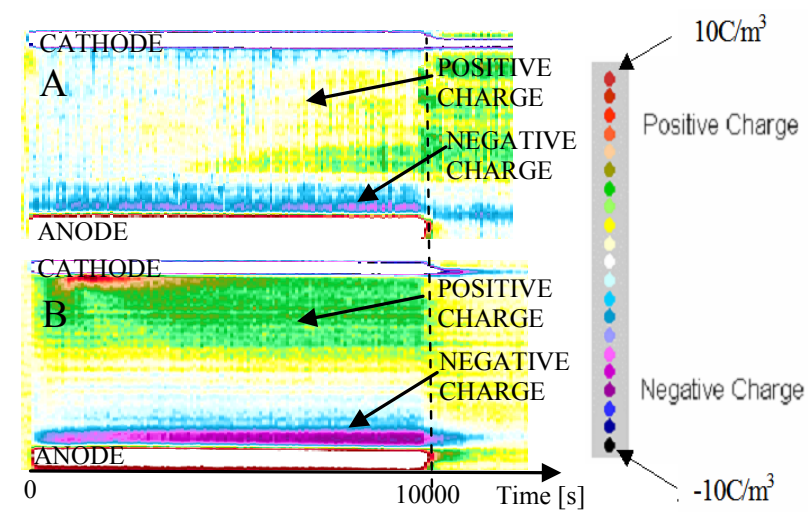

Figure 2. Space charge patterns obtained on mini-cable specimens, HVgrade XLPE. Poling voltage $=60 \mathrm{kV}(40 \mathrm{kV} / \mathrm{mm}$ average field $)$; Polarization duration $=10000 \mathrm{~s}$; Temperature $=25^{\circ} \mathrm{C}(\mathrm{A}), 70^{\circ} \mathrm{C}(\mathrm{B})$.

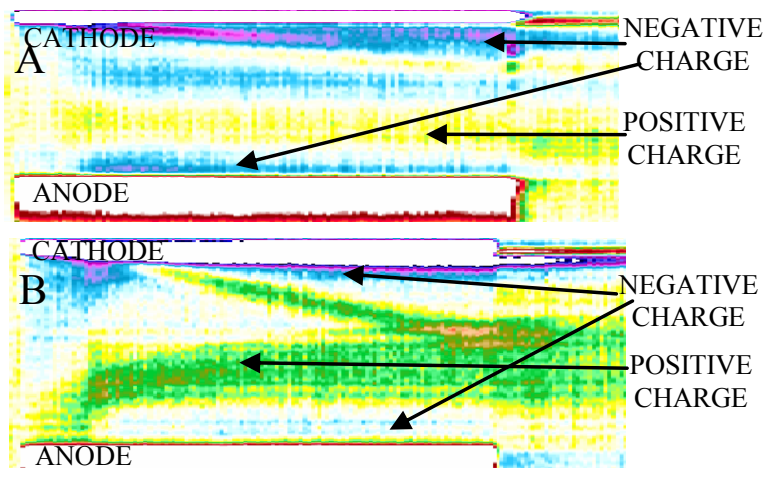

Figure 3. Space charge patterns obtained on mini-cable specimens, MV-grade XLPE. Poling voltage $=60 \mathrm{kV}(40 \mathrm{kV} / \mathrm{mm}$ average field $)$; Polarization duration $=10000 \mathrm{~s}$; Temperature $=25{ }^{\circ} \mathrm{C}$. Thermally treated (A) and untreated (B) specimens. The color scale is the same as in Fig. 2. 

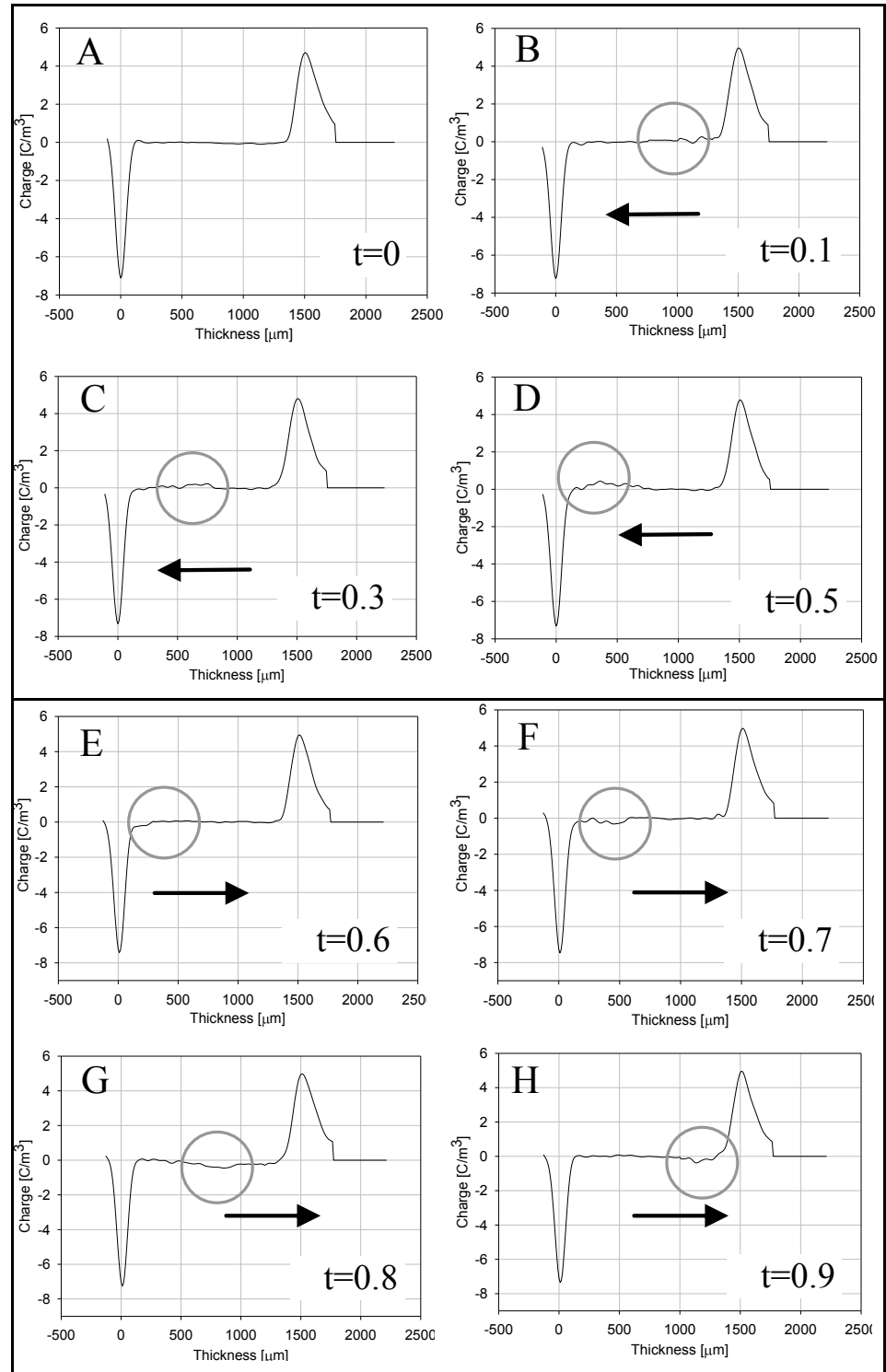

Figure 4. Evolution of charge profiles as a function of acquisition time, $t$ (indicated in the figures). Positive charge packet (A-D) and negative charge packet (E$\mathrm{H}$ ) are indicated by a circle. Test temperature $=35^{\circ} \mathrm{C}$. HV-grade XLPE.
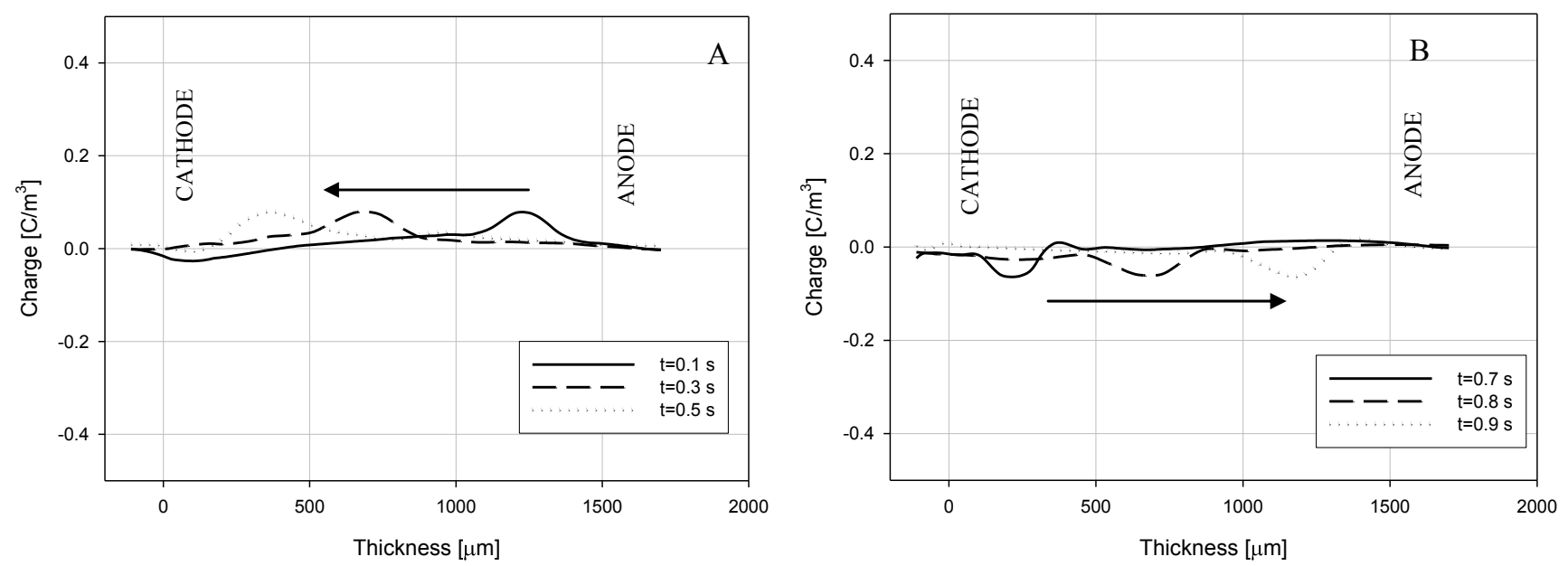

Figure 5. Transit of the positive (A) and negative (B) charge packets reported in Fig. 4, clarified by subtracting the profiles of the first acquisitions (Fig. 4A and $4 \mathrm{D}$, for positive and negative charge, respectively) from the charge profiles at time $t$, after de-noising by means of SWT. 
A further verification of the packet existence lies in its systematic dependence upon temperature, the fact that positive packets and negative packets possess different values of activation energy and repetition rate, and that the formation of heterocharge due to the injected charge packets can be described in terms of the packet size, repetition rate, and extraction rate constant. These features will be discussed in the subsequent Sections.

\section{DISCUSSION}

\subsection{EVIDENCE FOR FAST CHARGE PACKETS}

First of all, it must be pointed out that each charge packet (positive and negative) travelling from one electrode to the other involves only a small amount of charge (about 100-130 pC with a charge density of $0.07-$ $0.1 \mathrm{C} / \mathrm{m}^{3}$ ). However the packets are produced with a high repetition rate and therefore after some seconds from the beginning of polarization, several packets accumulate a noticeable amount of heterocharge close to both electrodes. This heterocharge buildup has been observed in our experiments only when a semiconducting layer is present between insulation and metal (copper or aluminium) electrode and for relatively-thick specimens. Tests performed on thin flat specimens having semicon layer only on one side showed, in fact, that no heterocharge was observed close to the electrode not in contact with the semicon [22]. It seems that semicon material can partially block the extraction of injected charge at the counter electrode. Thus, a heterocharge build-up may be observed close to the partially-blocking electrode $[9,22]$. If we assume that the extraction rate is proportional to the amount of heterocharge present (at least until space charge accumulation in the bulk is negligible) and that heterocharge accumulates because the rate of arrival of charge packets is initially greater than the rate of extraction, we obtain the following expression for the time-dependence of the heterocharge, $Q_{h e t}(t)$,

$$
Q_{\text {het }}(t)=\frac{2_{p a k} F}{k}(1-: \mathrm{xp}(-t))
$$

Here $Q_{h e t}(t)$ is the total heterocharge per square metre, $Q_{p a k}$ is the charge per square metre in a packet, $F$ is the packet repetition rate, and $k$ is the extraction rate constant. Since we measure $Q_{p a k}$ and $F$ independently of the heterocharge formation, equation (1) is completely defined if the saturation heterocharge quantity is known (i.e. $\left.Q_{p a k} F / k=Q_{h e t}(\infty)\right)$. A fit to the time dependence therefore gives an independent check to the value of the rate constant $k$ and provides support to the identification of the features shown in Figures 4 and 5 as charge packets. Figure 6 shows the results of such a fit for positive (Fig. 6A) and negative packets (Fig. 6B). The values of the parameters (average of 10 packets) of eq. (1) are: $F=2.5 \mathrm{~s}^{-1}, k=0.0625 \mathrm{~s}^{-1}, Q_{p a k}=1 \times 10^{-5} \mathrm{C} / \mathrm{m}^{2}$, for negative packets, $F=3.3 \mathrm{~s}^{-1}, k=0.0825 \mathrm{~s}^{-1}, Q_{\text {pak }}=2 \times 10^{-5}$ $\mathrm{C} / \mathrm{m}^{2}$, for positive packets. It can be seen that apart from a small displacement of the origin of the heterocharge buildup from $t=0$, the fit is very good.
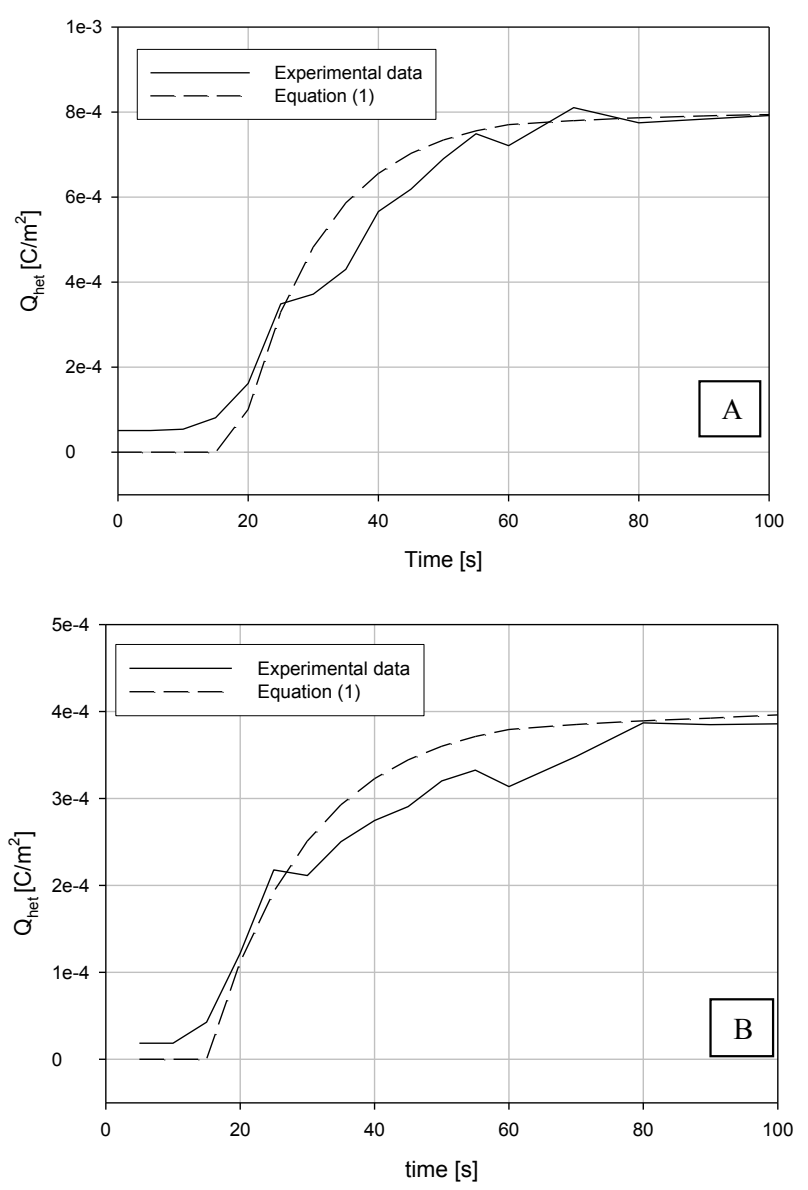

Figure 6. Positive (A) and negative (B) heterocharge build-up as a function of poling time from experimental data (thick line) and calculated by equation (1) (dashed line).

Another interesting piece of information one can get from the previous space charge profiles is that the time for the charge to cross the insulation bulk is very small, i.e., a fraction of a second, decreasing with increasing temperature. This could seem surprising for an insulating material having low conductivity such as XLPE. However, the energy of injected charge is large enough, considering the quite thin insulation ( $1.5 \mathrm{~mm}$ thick), to enable charge to reach the counter electrode in a very short time. It is noteworthy that the negative charge seems to be always faster than the positive charge at the same temperature. In order to provide a quantification of the charge dynamics, the average mobility value, $\mu$, of the charge packets (positive and negative) can be obtained roughly from $[24,25]$ :

$$
\mu=\frac{v}{E_{\text {mean }}}
$$

where $v$ is the speed of the charge packet; $E_{\text {mean }}$ is the average electric field applied to the insulation (in this case $=40 \mathrm{kV} / \mathrm{mm}$ ). Such a procedure was justified in [18] for slow charge packets where it was pointed out that the packet speed did not change with changes in field 
generated at the packet front, either as the packet crossed the insulation or in a packet sequence generated under the same applied field. The values of average mobility calculated according to eq. (2) are reported in Table 1. These values are at least 2-3 orders of magnitude larger than the typical values of apparent-trap-controlled mobility coming from slow packets (typically $10^{-12}-10^{-14}$ $\left.\mathrm{m}^{2} / \mathrm{Vs}\right)[8,26]$. Even using the local field in (2) the value of mobility is still very high. The average mobility of the fast packets increases with temperature, i.e. about 3 and 6 times larger on doubling the temperature (in ${ }^{\circ} \mathrm{C}$ ), for positive and negative charge packets, respectively. The mobility of negative charge is, thus, significantly larger than that relevant to positive charge, especially at high temperature (about 3 times larger at $70^{\circ} \mathrm{C}$ ).

Table 1. Average mobility values at three different temperatures for positive and negative fast charge packets.

\begin{tabular}{|c|c|c|c|}
\hline \multirow{2}{*}{} & \multicolumn{3}{|c|}{ Mobility $\left[\mathrm{m}^{2} / \mathrm{Vs}\right]$} \\
\cline { 2 - 4 } & $\mathbf{3 5}^{\circ} \mathbf{C}$ & $\mathbf{4 5}^{\circ} \mathbf{C}$ & $\mathbf{7 0}^{\circ} \mathbf{C}$ \\
\hline Positive charge & $7.2 \times 10^{-11}$ & $9.6 \times 10^{-11}$ & $1.9 \times 10^{-10}$ \\
\hline Negative charge & $9.6 \times 10^{-11}$ & $1.4 \times 10^{-10}$ & $5.7 \times 10^{-10}$ \\
\hline
\end{tabular}

The mobility of slow charge packets has been estimated from data at a single temperature to have large activation energies of the order of $1 \mathrm{eV}$ or more [27]. Measurement of the temperature-dependence of the transit time of slow packets [18] in XLPE material of the same origin as that used here yields an activation energy of $1.2 \mathrm{eV}$ for the mobility of positive packets, see Fig. 7. In order to determine whether the high mobility of the fast packets is associated with a substantially lower activation energy the mobility (log-values) reported in Table 1 are plotted as a function of $\mathrm{T}^{-1}(\mathrm{~T}=$ absolute temperature) according to the Arrhenius law (see Figures $8 \mathrm{~A}$ and $8 \mathrm{~B}$ for positive and negative charge packet mobility, respectively). The activation energy, $E_{a}$, is then calculated from the slope of the regression line: the larger the activation energy, the stronger the effect of temperature in increasing the mobility.

As can be seen from Fig. 8 the experimental data fit a straight line, meaning that the charge transport process follows the Arrhenius law, thus, providing a further confirmation that the charge packets highlighted here are real charges and not perturbations of the detected PEA signal due to noise. Moreover, the activation energy of negative charge is about 2 times larger than that relevant to positive charge, meaning that the dynamics of negative charges is much more affected by temperature than that relevant to positive charges. Furthermore the activation energies of both positive and negative fast packets are much lower than those appropriate to the slow packets, indicating that their high speed owes something to their low activation energies for transport.

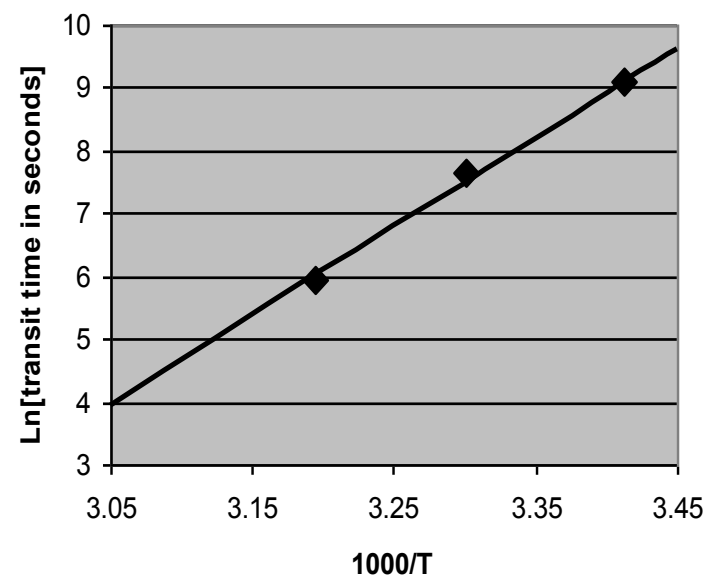

Figure 7. Arrhenius plot of logarithm of transit time for slow charge packets.
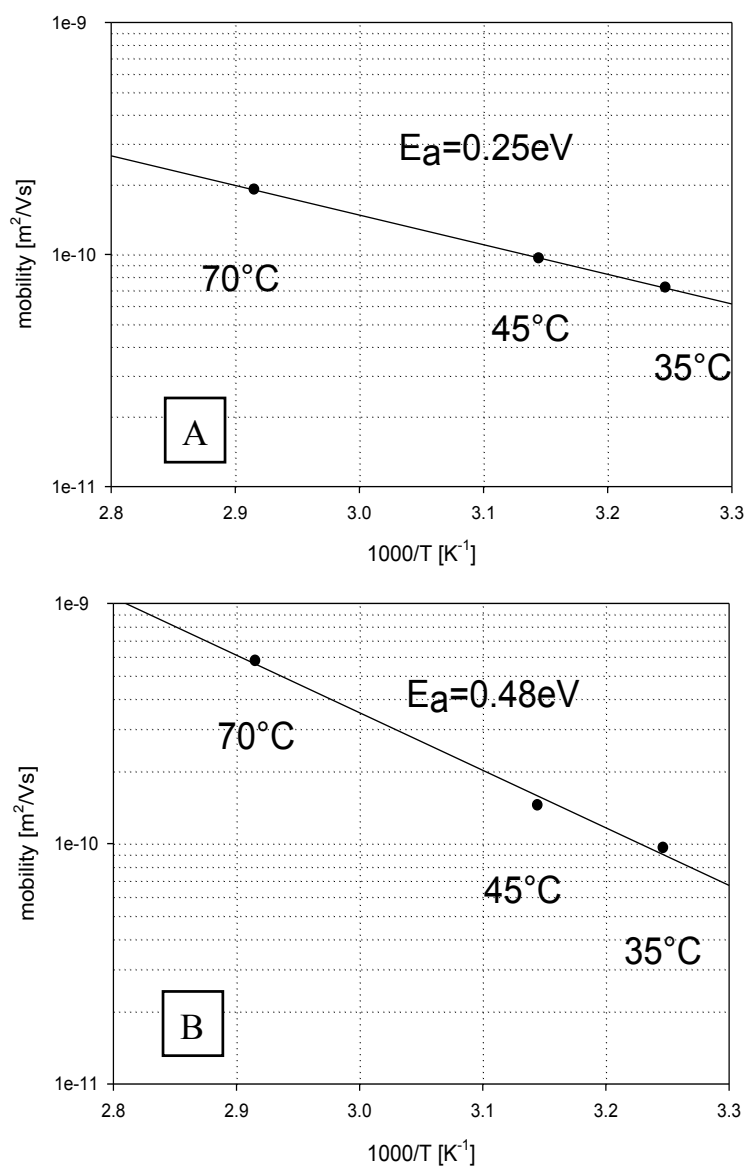

Figure 8. Arrhenius plot of mobility of positive (A) and negative (B) charge fast packets. The activation energy, $\mathrm{E}_{\mathrm{a}}$, is also indicated.

\subsection{CORRELATION BETWEEN FAST AND SLOW PACKETS}

The mobility of the slow packets reported in [18] was found to be $1.5 \times 10^{-16} \mathrm{~m}^{2} \mathrm{~V}^{-1} \mathrm{~s}^{-1}$ at $\mathrm{T}=293 \mathrm{~K}$, and was associated with charge residing in the deepest traps accessible in the narrow range of trap energies estimated from charge decay during depolarization at lower applied fields, see [28]. Simulations using an exponential trap 
energy distribution, [8], were able to reproduce features of the low-field charge distribution in similar XLPE peelings at $298 \mathrm{~K}$ with a charge mobility that goes from $10^{-14}$ to $10^{-13} \mathrm{~m}^{2} \mathrm{~V}^{-1} \mathrm{~s}^{-1}$, and up to $10^{-12}$ or higher for electrical fields larger than $50 \mathrm{kV} / \mathrm{mm}$, see Fig. 9. A comparison with the data of Table 1 and the mobility of the slow packets of Fig. 3, shows that the packets occasionally formed in the simulations of the model presented in [8] are of the slow type. Therefore, it seems that even when the trap depth range is extended to small values using the exponential distribution it is unable to reproduce the situation needed for the high mobility of fast charge packets. Although the simulations reported in [23] do not produce charge packets, they do define the conditions required for fast transit of the insulation sample and the accumulation of heterocharge. These are that: a) charge is injected into a state in which the charge has high mobility and, therefore, a small activation energy for transport, b) a very low or non-existent probability of trapping in deep traps, and c) a rate of charge extraction that is initially smaller than that of charge arrival in the vicinity of the extracting electrode. It can be seen that this picture is consistent with the measurements reported here, if features of the injection process cause the charge injection to take the form of pulses with the charge entering the high mobility state in low applied fields and the low mobility deep-trapped state in high fields. The constancy of the charge packet amplitude as it crosses the insulation implies that for fast packets the trapping rate and, hence, the establishment of a thermodynamic equilibrium distribution between the concentration of charges in the deep traps and in the shallow-trap high-mobility state, is very much slower than the time over which the experiment takes place. A lack of interchange between the deep-trap states and the high-mobility states is also required for the existence of slow charge packets, since in this case one would expect the high applied field to promote a considerable portion of the injected charge into the shallower states, which would reduce the packet amplitude in a way that is not observed. This inability to transfer charge between the two classes of states indicates that the association of deep traps with chemical contaminants and shallow traps with physical traps $[29,30]$ is probably over-simplistic, and it is noteworthy that the activation energy for the mobility of fast negative packets is twice that estimated for physical electron traps.

An exciting interpretation of the results relevant to fast packets can be driven considering that they involve likely electronic carriers. The negative packets, in fact, could consist of electrons moving in the interchain space (remembering the negative electron affinity of PE [31]) thanks to chain relaxation mechanism which contributes to charge motion. The positive packets could be likely formed by holes, easily moving along the chains and tunnelling from one chain to another, again thanks to chain motion. This picture would explain, in fact, the different mobility detected experimentally in positive and negative packets and why the associated charge does not seem to recombine in the bulk (positive and negative charges follow different paths). Moreover, if the packet movement is due to chain relaxation, charge hopping through a quasi-continuous distribution of localized states seems not to apply to the movement of fast packets and this justifies the very narrow trapping level for positive $(0.25 \mathrm{eV})$ and negative $(0.48 \mathrm{eV})$ charges. Anyway, the nature of charge transport in packets as well as the reason why packets remain as packets and do not spread out, particularly in the case of the slow packets whose transit may take hours even in thin $150 \mu \mathrm{m}$ samples, requires a detailed analysis of the transport mechanism that will be reported in a subsequent paper. What does seem clear is that the explanation of charge packets as the manifestation of a peak in the dependence of the charge carrier speed as a function of increasing electric field, [15], cannot be accepted as the packet does not slow down when the field at the packet front increases, either during transit of the sample or in a packet sequence $[10,18]$. Nor do the packets speed up when the packetfront field diminishes in a slow charge packet sequence.

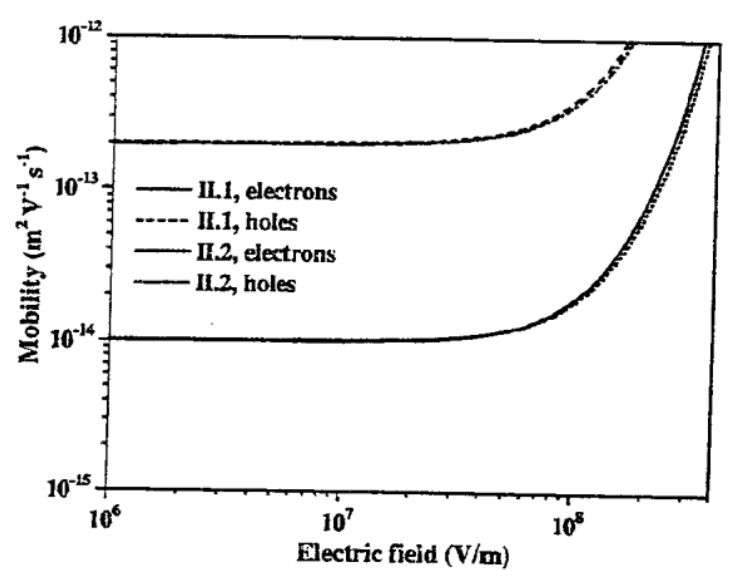

Figure 9. Electron and hole mobility as a function of electric field for two sets of parameters (II.1 and II.2) from the mobility model discussed in [8]. The associated data are taken from [8].

The conditions at the interface with the injecting electrode clearly play a role in causing injection to take the form of pulses leading to charge transit as a packet, and the interface field must also be influential in controlling the type of state into which the injection is made. These considerations imply that different electrode materials may behave differently. They also suggest that the way that various chemical species facilitate charge packet formation is via their influence upon interface states and the interface morphology. It has been shown [10] that the inception of slow charge packets requires the interface field to exceed a threshold value. This field is high enough $(150 \mathrm{kV} / \mathrm{mm}$ in [10]) to allow the possibility of such a massive charge injection that the interface field is reduced to a value below the threshold level. It could be speculated that depending on applied voltage and field, and for insulation thicknesses large enough to force charge carriers to be subjected to multiple trapping/detrapping processes, fast packets are needed to bring enough heterocharge to the counter-electrode such 
as to magnify the interface field to the required value. This does not seem to be the case in [10] where a region of negative homo-charge takes several hours to spread across the sample and provide the hetero-charge field enhancement required to initiate positive packets from the anode. However it may occur in other cases. If the applied field/temperature is large enough, or contamination is noticeable (Fig. 3), the interface field can generate directly a slow packet, without needing fast packets to accumulate heterocharge. Fast packets may still exist, but it is very difficult to observe them due to the small amount of charge associated with them. It should be pointed out that we would not expect fast charge packets to contribute significantly to the measured conductivity because they carry such small amounts of charge and the conductivity is usually measured from the current in the quasi-steady state after many hours of polarization. They or their associated high-mobility states may however be involved in charge re-distribution produced by thermal gradients in low field [32].

\section{CONCLUSIONS}

The results presented in this paper provide a novel explanation of the very rapid heterocharge formation which is often observed in HVDC cables and cable models. Instead of the separation of ionic species under the action of an electric field, usually adopted to explain such behaviour, experimental tests carried out at high sampling rate show that in a fraction of a second charge packets are injected at the electrodes and cross the insulation, showing a mobility considerably higher than that expected according to literature and derived from conduction current or conventional space charge measurements. These charge packets are small in magnitude and are generated at fields well below those typically needed for slow packet generation. The mobility of the fast charge packets may be orders of magnitude larger than those of the slow packets, which may in some part be ascribed to the much lower activation energies of the fast packets. Both positive and negative fast packets are observed, with different activation energies and repetition frequencies. Hetero-charge is accumulated because semicon counter electrodes are initially unable to extract charge at the rate at which it arrives in their vicinity. A dynamic equilibrium is eventually set up in a time depending upon the extraction rate constant, and with a saturation charge quantity that is dependent upon the charge packet magnitude and repetition frequency as well as the extraction rate constant. The quantity of heterocharge is different for positive and negative polarities. Our results imply that there are two modes of charge transport, one operative in fast packets and one in slow packets, and that these transport modes only interchange charge very slowly if at all. This research confirms that investigation of fast space chargeassociated processes in polymeric insulation is an approach that must be promoted to understand better the mechanisms of space charge formation.

\section{ACKNOWLEDGMENTS}

Part of the research discussed in this paper was performed within the European Project (of the $5^{\text {th }}$ Framework-program) HVDC, "Benefits of HVDC Links in the European Power Electrical System and Improved HVDC Technology" (Contract No. ENK6-CT-200200670). One of us (Leonard A. Dissado) also thanks the Institute of Advanced Study (ISA) of the University of Bologna for a Senior Fellowship during the preparation of this paper.

\section{REFERENCES}

[1] G.C. Montanari, "The electrical degradation threshold of polyethylene investigated by space charge and conduction current measurements", IEEE Trans. on Diel. El. Insul., Vol. 7, No. 3, pp. 309-315, June 2000.

[2] T. Takada, "Acoustic and Optical Methods for Measuring Electric Charge Distribution in Dielectrics", IEEE Trans. on Diel. El. Insul., Vol. 6, pp. 519-547, 1999.

[3] M. Abou-Dakka, A. Bulinski, S. Bamji, "Space charge development and breakdown in XLPE under DC field", IEEE Trans. on Diel. El. Insul., Vol. 11, pp. 41-49, 2004.

[4] G. C. Montanari, "Dielectric material properties investigated through space charge measurements", IEEE Trans. on Diel. El. Insul., Vol. 11, pp. 56-64, 2004.

[5] T. Takada, M. Kojima, Y. Tanaka, Y. Ohki, "Observation and numerical calculation of space charge behavior in gammairradiated low density polyethylene", IEEE ICSD, Leicester, UK, July 1995, pp. 274-278.

[6] C. Inguimbert, Y. Carrere, V. Griseri, B. Dirassen, L. Levy, D. Payan, K. Fukunaga, "Measurement and modeling of charge profiles in irradiated dielectrics", IEEE ICSD, Toulouse, France, July 2004, pp. 951-954.

[7] S. Le Roy, P. Segur, G. Teyssedre and C. Laurent, "Description of bipolar charge transport in polyethylene using a fluid model with a constant mobility: model prediction", Journ. Phys. D: Appl. Phys. Vol. 37, pp. 298-305, 2004.

[8] F. Boufayed, G. Teyssedre, C. Laurent, S. Le Roy, L.A. Dissado, P. Segur, G.C. Montanari, "Models of bipolar charge transport in polyethylene", Journ. Appl. Phys., Vol. 100, pp. 104105.1-10, 2006.

[9] D. Fabiani, G.C. Montanari, C. Laurent, G. Teyssedre, P.H.F, Morshuis, R. Bodega, L.A. Dissado, A. Campus, U.H. Nilsson, "Polymeric HVDC cable design and space charge accumulation. Part 1: insulation/semicon interface", IEEE Electr. Insul. Magazine, Vol. 23, No. 6, pp. 11-19, 2007

[10] A. See, L. A. Dissado, J. C. Fothergill, "Electric field criteria for charge packet formation and movement in XLPE", IEEE Trans. on Diel. El. Insul., Vol. 8, pp. 859-866, 2001.

[11] F. Zheng; Y. Zhang; C. Wu; L. Hu; Z. Xia, "Space charge packet behavior in low density polyethylene" IEEE ICPADM, Nagoya, Japan, June 2003, pp. 681-684.

[12] M. Fukuma, K. Fukunaga, C. Laurent, "Packet-like space charge in polyethylene probed with a 2D-spatial resolution" IEEE CEIDP, Kansas City, MO (USA), October 2006, pp.748-751.

[13] K. Kaneko, T. Odaka, Y. Suzuoki, T. Mizutani, "Study on space charge dynamics by computer simulation: Formation and transport of space charge packet", IEEE ICPADM, Seul, Korea, 1997, pp. 821-824.

[14] H. Kon, Y. Suzuoki, T. Mizutani, M. Ieda, N. Yoshifuji, "Packetlike space charges and conduction current in polyethylene cable insulation", IEEE Trans. on Diel. El. Insul., Vol. 3, pp. 380-385, 1996. 
[15] J.P. Jones, J.P. Lewellyn, T.J. Lewis, "The contribution of fieldinduced morphological change to the electrical aging and breakdown of Polyethylene", IEEE Trans. on Diel. El. Insul., Vol. 12, pp. 951-966, 2005.

[16] K. Matsui, Y. Tanaka, T. Takada, T. Fukao, K. Fukunaga, T. Maeno, J. M. Alison, "Space charge behavior in low density polyethylene at pre-breakdown", IEEE Trans. on Diel. El. Insul., Vol. 12, pp. 406-415, 2005

[17] Y. Zhang, J. Lewiner, C. Alquié and N. Hampton, "Evidence of strong correlation between space charge build up and breakdown in cable insulation", IEEE Trans. on Diel. El. Insul., Vol. 3, pp. 778-783, 1996.

[18] L. A. Dissado, S. Zadeh, J. C. Fothergill, A. See, "Temperature dependence of charge packet velocity in XLPE cable peelings", IEEE CEIDP, Vancouver, Canada, October 2007, pp. 425-428.

[19] N. Hozumi, H. Suzuki, T. Okamoto, K. Watanabe, A. Watanabe, "Direct observation of time dependent space charge profiles in XLPE cable under high electric fields", IEEE Trans. on Diel. El. Insul., Vol. 1, pp. 1068-1076, 1994.

[20] N. Hozumi, T. Takada, H. Suzuki, T. Okamoto, "Space charge behavior in XLPE cable insulation under $0.2-1.2 \mathrm{MV} / \mathrm{cm} \mathrm{dc}$ fields", IEEE Trans. on Diel. El. Insul., Vol. 5, pp. 82-90, 1998.

[21] K. Kaneko, T. Mizutani, Y. Suzuoki, "Computer simulation on formation of space charge packets in XLPE films", IEEE Trans. on Diel. El. Insul., Vol. 6, pp. 152-158, 1999.

[22] D. Fabiani, G. C. Montanari, R. Bodega, L. A. Dissado, C. Laurent, G. Teyssedre, A. Campus, U. H. Nilsson, P.H.F. Morshuis, "Effect of semicon/insulation interface on space charge formation in HVDC polymeric cables", JICABLE, Versailles, France, June 2007, pp. 470-473.

[23] S. Le Roy, G.Teyssedre, C.Laurent, L.A..Dissado, G.C.Montanari, "Relative importance of trapping and extraction in the simulation of space charge distribution in polymeric insulators under DC potential", IEEE ICSD’07, Winchester, UK, July 2007, pp. 494497.

[24] D. A. Seanor, Electrical Properties of Polymers, Academic Press, London, 1982.

[25] G. Mazzanti, G.C. Montanari, F. Palmieri, J. Alison, "Apparent trap-controlled mobility evaluation in insulating polymers through depolarisation characteristics derived by space charge measurements", Journ. Appl. Phys., Vol. 94, No. 9, pp. 59976004, November 2003.

[26] S. Leroy, F. Boufayed, F. Baudoin, G. Teyssedre, C. Laurent, "A user-friendly tool for simulating the time-dependent field distribution in PE insulation on the basis of a physical approach", JICABLE, Versailles, France, June 2007, pp. 714-718.

[27] G. Mazzanti, G.C. Montanari, J. M. Alison, “A space-charge based method for the estimation of apparent mobility and trap depth as markers for insulation degradation. Theoretical basis and experimental validation", IEEE Trans. on Diel. El. Insul., Vol. 10, pp. 187-197, 2003.

[28] A.Tzimas, M.Fu, L.A.Dissado, "Characterization of electrothermally aged XLPE cable peelings through space charge measurements", IEEE CEIDP, Nashville, USA, October 2005, pp. 30-33.

[29] M. Meunier, N. Quirke, A. Aslanides, "Molecular modeling of electron traps in polymer insulators: Chemical defects and impurities", Journ. Chem. Phys., Vol. 115, pp. 2876-2881, 2001.

[30] J. A. Anta, G. Marcelli, M. Meunier, N. Quirke, "Models of electron trapping and transport in polyethylene: Current-voltage characteristics", Journ. Appl. Phys., Vol. 92, pp. 1002-1008, 2002.

[31] S. Serra, E. Tosatti, S. Iarlori, S. Scandolo, and G. Santoro "Interchain electron states in polyethylene, Phys. Rev. B, Vol. 62, pp. 4389-4393, 2000.

[32] K. R. Bambery, R. J. Fleming and J. T. Holboll, "Space charge profiles in low density polyethylene samples containing a permittivity/conductivity gradient", Journal of Physics D: Appl. Phys. Vol. 34, pp. 3071-3077, 2001.

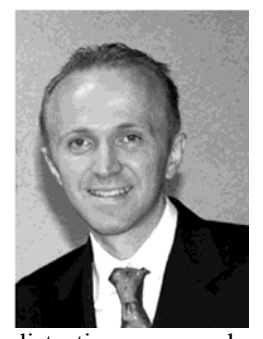

Davide Fabiani (M'98) was born in Forlì, Italy, on 7 January 1972 . He received the M. Sc. (honors) and $\mathrm{PhD}$ degrees in Electrical Engineering from the University of Bologna in 1997 and 2002, respectively. He is assistant professor at the Department of Electrical Engineering of the University of Bologna since 2005 where he teaches the course of "Modelling and Engineering of Electrical Materials". His research interests deal with the effect of voltage distortion on accelerating insulation degradation, characterization of insulating, magnetic, superconducting, nanocomposite and electret materials, aging investigation and diagnosis of power system insulation and, particularly, motor windings subjected to fast repetitive pulses. To date he is author or co-author of more than 80 papers. He is a member of IEEE DEIS, IEEE PES and AEI. He is co-founder of the University spin-off TechImp Spa.

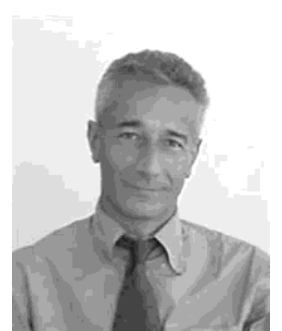

Gian Carlo Montanari (M'87-SM'90-F'00) was born on $8 / 11 / 55$. In 1979 , he took the Master degree in Electrical Engineering at the University of Bologna. He is currently Full Professor of Electrical Technology at the Department of Electrical Engineering of the University of Bologna, and teaches courses of Technology and Reliability. He has worked since 1979 in the field of aging and endurance of solid insulating materials and systems, of diagnostics of electrical systems and innovative electrical materials (magnetics, nanomaterials, electrets, superconductors). He has been also engaged in the fields of power quality and energy market, power electronics, reliability and statistics of electrical systems. He is IEEE Fellow and member of AEI and Institute of Physics. He is member of the AdCom of the IEEE DEIS. Since 1996 he is President of the Italian Chapter of the IEEE DEIS. He is convener of the Statistics Committee and member of the Space Charge, Multifactor Stress and Meetings Committees of IEEE DEIS. He is Associate Editor of IEEE Transactions on Dielectrics and Electrical Insulation. $\mathrm{He}$ is founder and President of the spin-off TechImp, established on 1999. He is author or coauthor of about 550 scientific papers.

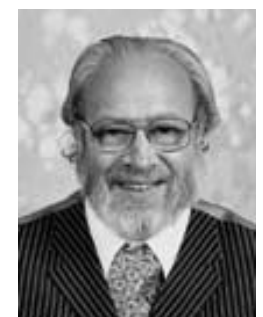

Leonard A. Dissado (SM'96-F'06) graduated from University College London with a degree in chemistry in 1963, obtained a Ph.D. degree in theoretical chemistry in 1966 and a D.Sc. degree in 1990 from the same university. In 2007 he was honoured by the Universite Paul Sabatier, Toulouse, with the award of a Doctuer Honoris Causa. After rotating between Australia and England twice, he settled in Chelsea College in 1977 to carry out research in dielectrics. Since then he has published many papers and one book, together with John Fothergill, on breakdown and associated topics. In 1995 he moved to the University of Leicester and was promoted to professor in 1998. He has been a visiting professor at the University Pierre and Marie Curie in Paris, Paul Sabatier University in Toulouse, and Nagoya University, and a Senior Visiting Fellow at the Institute of Advanced Studies of the University of Bologna. He also has given numerous invited lectures, including the Whitehead Memorial Lecture in 2002. Currently he is an associate editor of the IEEE Transactions on DEI, co-chair of the Multifactor Aging Committee of DEIS, and a member of DEIS AdCom. 


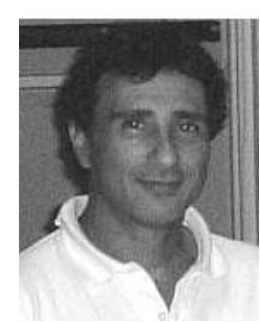

Christian Laurent (M'98, S'07) was born in Limoges, France, in 1953. He studied solid state physics at the National Institute for Applied Sciences in Toulouse and received his Eng. degree in physics in 1976. He joined the Electrical Engineering Laboratory at Paul Sabatier University in 1977 to study electrical treeing and partial discharge phenomena, which were the topics of his Dr. Eng. Degree (1979). He joined CNRS (National Centre for Scientific Research) in 1981 and got his Doc-ès Sc. Phys. in 1984. In 1985, he spent one year as a post-doctoral fellow with the IBM Almaden Research Center, where he studied plasma-polymerized thin films. Back in Toulouse he developed an approach to electrical ageing in polymeric materials based on luminescence analysis. He is now dealing with experimental and modeling activity relating to charge transport and ageing. He is currently Research Director at CNRS and Director of the Laboratory of Plasma and Energy Conversion -LAPLACE- in Toulouse.

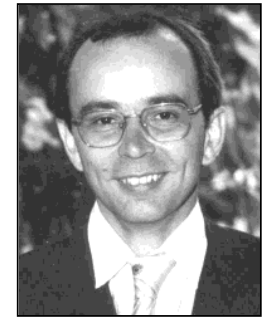

Gilbert Teyssedre was born in May 1966 in Rodez, France. He received his Engineer Degree in materials physics in 1989 from the National Institute for Applied Science (INSA) and graduated in solid state physics the same year. Then he joined the Solid State Physics $\mathrm{Lab}$ in Toulouse and obtained a PhD degree in 1993 for a work on transition phenomena and electro-active properties of fluorinated ferroelectric polymers. He entered the CNRS in 1995 and has been working since then at the Electrical Engineering Lab (now LAPLACE) in Toulouse. His research activities concern the development of luminescence techniques in insulating polymers with focus on chemical and physical structure, degradation phenomena, space charge and transport properties. He is currently Research Director at CNRS and is leading a team working on the reliability of dielectrics in electrical equipment. 